\title{
Evaluation of pediatric patients presenting with vertigo
}

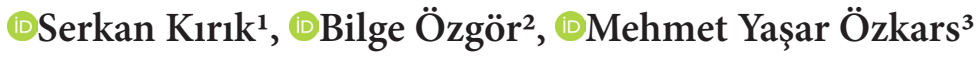 \\ ${ }^{1}$ Kahramanmaraş Sütçü İmam University, Faculty of Medicine, Department of Pediatrics, Division of Pediatric Neurology, Kahramanmaraş, Turkey \\ 2İnönü University, Faculty of Medicine, Department of Pediatrics, Division of Pediatric Neurology, Malatya, Turkey \\ ${ }^{3}$ Kahramanmaraş Sütçü İmam University, Faculty of Medicine, Department of Pediatrics, Kahramanmaraş, Turkey
}

Cite this article as: Kırık S, Özgör B, Özkars MY. Evaluation of pediatric patients presenting with vertigo. J Health Sci Med 2021; 4(3): $247-251$.

\begin{abstract}
Objective: Vertigo in children is a less studied subject than that in the adulthood. The aim of the present study was to determine the clinical characteristics of children presenting to the pediatric neurology clinic with vertigo.

Material and Method: Fourty children were enrolled. The patients digital medical datas analyzed retrospectively. The study included all patients younger than 18 years of age who presented to Kahramanmaras Sütçü İmam University Hospital and Aydın Maternity and Children's Hospital, Pediatric Neurology Outpatient Clinic between July 2017 and July 2020.

Results: In our study, 40 patients with a mean age of vertigo onset between 2 years and 17 years were evaluated. The most common complaints accompanying vertigo were headache (57.5\%) and nausea (35\%). The most commonly detected clinical cause of vertigo was migraine associated vertigo (MAV) $(n=18)$; twelve patients had psychogenic vertigo, 3 patients had orthostatic hypotension $(\mathrm{OH})$, and 7 patients had benign childhood paroxysmal vertigo (BCPV). While the most common cause of vertigo among children under the age of 6 was BCPV, MAV was the most common etiology among children above the age of 6. It was observed that headache more commonly accompanied vertigo in patients with MAV $(p<0.001)$. Vertigo episodes longer than five minutes were less common in patients with BCPV and OH. Symptomatic worsening occurred in children diagnosed with BCPV and MAV.

Conclusion: In the majority of pediatric patients with vertigo, a detailed examination including a detailed history and neurological and audio logical evaluations is sufficient for diagnosis. Considering the anxiety of families in this patient group, especially in the pre-school age group, appropriate approach, as well as prevent unnecessary tests.
\end{abstract}

Keywords: Child, migraine, vertigo, benign paroxysmal vertigo

\section{INTRODUCTION}

Vertigo is commonly defined as a patient's perception that he/she is rotating relative to his/her environment or his/her environment is rotating around him/her. Vertigo is an uncommon symptom among children and adolescents. The duration of vertigo is important in terms of its adverse effects on both the affected person and his/her family $(1,2)$. The prevalence of vertigo in the childhood period has been reported to be $5.3 \%$. Vertigo in the childhood age group is different than that in the adulthood depending on the description of symptoms and causes (such as migraine, arteritis etc.), developmental stage of balance and vestibular functions, and causes of vertigo. In many studies, migraine associated vertigo (MAV) and benign childhood paroxysmal vertigo (BCPV) have been named as the most common causes of vertigo. While BCPV is the most common cause of vertigo in pre-school children, MAV is a more common etiology in adolescence (1-5).
The most critical steps in the diagnosis are detailed history taking and physical examination. As young children cannot adequately describe their complaints, a family's observations are of great significance. History of complaints, neurological examination, and otological tests, many patients are diagnosed without the need to perform further tests. The most important conditions that must be considered in the differential diagnosis are posterior fossa masses, epileptic seizures and vestibular disorders. BCPV is characterized by benign, selflimiting, recurrent vertigo attacks of short duration that occur without any stimulus in healthy children. It was described as an episodic condition related with migraine. The patients recover spontaneously $(2,3)$. Having at least 5 vertigo attacks is one of the diagnostic criteria $(6,7)$. Patients are completely normal in the inter-attack periods. 
Headache is the common complaint in children and adolescents presenting with vertigo. The co-existence of migraine headache and vestibular vertigo is quite common in the community. MAV is more common in children than adults (8-11). Psychogenic (somatoform) vertigo may occur as a subjective complaint in psychiatric disorders. It is more commonly associated with anxiety, depression, and behavioral disorders. There are few systematic studies on psychogenic vertigo in children and adolescents. These patients present with chronic vertigo, and their physical examination and vestibular tests are normal. It is more common in girls. There is no balance problem; the onset of symptoms with the emergence of the triggering event is typical $(2,3,12,13)$.

The aim of this study was to determine the etiology and clinical features of vertigo in children and adolescents, and to contribute to the establishment of a general approach to this symptom by defining its clinical characteristics.

\section{MATERIAL AND METHOD}

We retrospectively collected electronic data of patients under 18 years old who admitted with vertigo to Kahramanmaraş Sütçü İmam University Hospital and Aydın Maternity and Children's Hospital, Department of Pediatric Neurology clinic between July 2017 and July 2020. Approval for the study was granted by the Ethics Committee of Kahramanmaraş Sütçü İmam University Medical Faculty (Date: 15.03.2017, session no: 2017/04, Decision No: 43). All procedures were carried out in accordance with the ethical rules and the principles of the Declaration of Helsinki. Children were evaluated with variables including gender, age at vertigo onset, accompanying symptoms, history trauma, emergence or worsening of symptoms during travel (motion sickness), comorbidities, family history of vertigo and migraine, epilepsy, neurologic examination findings, imaging results, vital signs including blood pressure, electroencephalography (EEG) results, and the final diagnosis were recorded.

Benign childhood paroxysmal vertigo is actually a diagnosis of exclusion of probable reasons of vertigo. In BCPV attacks are brief and severe. Child has normal physical and neurological examination between attacks. In most cases, for the differential diagnosis (intracranial tumors etc.) magnetic resonance imaging (MRI) and EEG performed. The diagnosis of MAV was made if the patient had migraine headaches following or accompanying vertigo attacks. Usually family history of migraine existing. The diagnosis of psychogenic (somatoform) vertigo was made in cases with normal neurological and audiological examination who described association of attacks with stressful situations, or if no etiology could be explained by neurological, vestibular examinations, MRI, and EEG. ICHD 3Beta (Benign paroxysmal childhood vertigo (ICHD III beta 1.6.2) diagnostic criteria were used to diagnose both BCPV and MAV $(6,7)$. In addition, the patients were evaluated for other possible disorders by the department of child psychiatry. The exclusion criteria included having intracranial space-occupying lesions, epilepsy, intracranial bleeding, and missing medical records.

\section{Diagnostic criteria of BCPV (6):}

A. At least five attacks fulfilling criteria $\mathrm{B}$ and $\mathrm{C}$

B. Vertigo occurring without warning, maximal at onset and resolving spontaneously after minutes to hours without loss of consciousness

C. At least one of the following five associated symptoms or signs:

1. Nystagmus

2. Ataxia

3. Vomiting

4. Pallor

5. Fearfulness

D. Normal neurological examination and audiometric and vestibular functions between attacks

E. Not attributed to another disorder.

\section{Diagnosis of MAV $(6,7)$}

- At least 5 episodes of vestibular symptoms of moderate or severe intensity lasting 5 minutes to 72 hours

- Current or previous history of migraines with or without aura according to the ICHD classification

- One or more of the following migraine features with at least $50 \%$ of vestibular episodes:

1.Headache with at least 2 of the following characteristics

2.One-sided location, pulsating quality, moderate or severe pain intensity; photophobia or phonophobia

\section{Visual aura}

- Not better accounted for by another vestibular or ICHD diagnosis

\section{Statistical Analysis}

Descriptive statistics included median, mean, percentage, and standard deviation. SPSS 22 statistical software package was used for statistical analyses. Kruskal-Wallis test was used to determine among groups differences. $\mathrm{P}$ value $<0.05$ was considered statistically significant. 


\section{RESULTS}

The number of patients presenting with vertigo was 53 . Nine patients were excluded due to reasons including intracranial space-occupying lesion $(n=5)$, epilepsy $(n=2)$, and intracranial bleeding $(n=4)$. As there was no trauma emergency unit at our clinic, no patient with traumainduced vertigo was present. In addition, 4 patients with mental retardation were excluded. The study enrolled 40 patients in total. The number of female patients was 17 $(\mathrm{F} / \mathrm{M}=1.35)$. The mean age of the patients was $11.46 \pm 3.92$ years and the median age was 10.2 years (20 months-17 years). The most commonly diagnosed disorder was MAV ( $n=18) ; 12$ patients had psychogenic vertigo, and 7 patients had BCPV. The patients were divided into two groups as those younger than the age of 6 and those older than the age of 6 . The number of patients under the age of 6 was $8(20 \%)$, and the number of patients above the age of 6 was $32(80 \%)$. BCPV was predominantly diagnosed in the patients under the age of $6(n=6)$. Psychogenic vertigo (n:12) had the highest percentage in girls ( 9 female $(75 \%) / 3$ male $(25 \%))$ than in the other groups. Eleven of the patients with the diagnosis of psychogenic vertigo were older than 12 years. The age range and the diagnoses of the patients are shown on Table 1.

Three patients had vertigo associated with orthostatic hypotension. Except for orthostatic hypotension in three patients, other vital signs (respiratory rate, body temperature, pulse rate) were normal in all patients.

The most common symptoms were headache (57.5\%) (18 of them had MAV, 3 psychogenic vertigo, and $2 \mathrm{BCPV}$ ), nausea (35\%) (12 of them had MAV, $2 \mathrm{BCPV}$ ), vomiting (30\%) (10 had MAV, 2 BCPV), and pallor (27.5\%) (7 had BCPV, $3 \mathrm{OH}, 1 \mathrm{MAV})$. The most common symptoms in patients with MAV were headache and nausea. The most common sign in patients with BCPV was pallor. Nystagmus was present only in patients diagnosed with BCPV $(n=3)$ (Table). Family history of migraine was significantly more common in patients with MAV and BCPV than patients with psychogenic vertigo and orthostatic hypotension $(\mathrm{p}<0.001)$.
Ten patients with MAV had a vertigo duration of $<5$ minutes while 8 had a vertigo duration of 5-30 minutes. Vertigo duration was $<5$ minutes in all patients with vertigo while it was $<1$ minute in 8 of them. Eight patients diagnosed with psychogenic vertigo had a vertigo duration of $<5$ minutes while 3 of them had a vertigo duration of 5-30 minutes, and 1 of them had a vertigo duration of $>30$ minutes. All patients with $\mathrm{OH}$ had a vertigo duration of $<5$ minutes. The duration of attacks was significantly shorter in patients with $\mathrm{BCPV}$ and $\mathrm{OH}$ than the other groups $(p<0.001)$. Attack frequency at the time of admission was significantly greater in psychogenic vertigo $(8.27 \pm 3.12)$ and MAV (7.35 \pm 4.16$)$ compared to the other groups $(\mathrm{p}<0.001)$. It was found that some patients with MAV and BCPV (38.8\% and $57.1 \%$, respectively) frequently had symptoms that emerge during or are exacerbated by travel. No correlation between symptoms and travel was reported by patients with psychogenic vertigo or $\mathrm{OH}(\mathrm{p}<0.001)$.

All of forty patients had an electroencephalogram (EEG). Epileptiform discharge was not observed in any of the patients. Sixteen patients had an electrocardiogram (ECG). No cardiac arrhythmia was observed in any of them. Vertigo episodes in patients with $\mathrm{OH}$ were related to $\mathrm{OH}$ by a pediatric cardiologist.

All patients were evaluated by an otorhinolaryngology specialist. None of them had hearing loss. All patients underwent neuroimaging (32 patients underwent magnetic resonance imaging (MRI), 8 patients computerized tomography (CT)). The most commonly detected sign was an arachnoid cyst (6 patients). There was no significant difference between patients with and without arachnoid cysts in terms of vertigo duration and attack frequency $(\mathrm{p}=0.61)$.

\section{DISCUSSION}

Vertigo of childhood can prove difficult to diagnose, especially in young children with limited ability to describe their symptoms. Vertigo may present before, during, or without headache. It is more common in children than adults. The two most common conditions

\begin{tabular}{|c|c|c|c|c|}
\hline & BCPV & MAV & Psychogenic vertigo & $\mathrm{OH}$ \\
\hline $\mathrm{F} / \mathrm{M}(\mathrm{n} / \mathrm{n})$ & $3 / 4$ & $9 / 9$ & $9 / 3$ & $1 / 2$ \\
\hline Age (mean) & $3.39 \pm 2.86$ & $9.52 \pm 4.16$ & $14.51 \pm 3.87$ & $12.65 \pm 3.4$ \\
\hline Attack duration(min) (mean) & $2.56 \pm 1.14$ & $7.9 \pm 5.67$ & $6.73 \pm 4.71$ & $2.12 \pm 2.6$ \\
\hline Family history for migraine (\%) & $85.7 \%$ & $77.7 \%$ & $16.6 \%$ & 0 \\
\hline Attack frequency/month (mean) & $3.51 \pm 2.28$ & $7.35 \pm 4.16$ & $8.27 \pm 3.12$ & $4.18 \pm 3.42$ \\
\hline Headache (\%) & $28.5 \%$ & $100 \%$ & $26 \%$ & 0 \\
\hline Nausea (\%) & $2(28.5 \%)$ & $12(66.6 \%)$ & 0 & 0 \\
\hline Pallor (\%) & $100 \%$ & $5.5 \%$ & 0 & $100 \%$ \\
\hline Nystagmus (\%) & $42.8 \%$ & 0 & 0 & 0 \\
\hline Motion sickness (\%) & $57.1 \%$ & $38.8 \%$ & 0 & 0 \\
\hline
\end{tabular}


seen in our study were MAV and psychogenic vertigo. Many studies to date have reported varying shares of different etiological conditions $(1,4,12)$. In a study by Batu et al. (3) the two most common diagnoses were BCPV and psychogenic vertigo while MAV was reported as the fifth most common cause. It was considered that the expressibility of complaints related to age group and our study center being a second step institution were the possible causes of this finding. Due to the absence of other pediatric clinics (pediatric gastroenterologycardiology etc.), patient diversity was less than in tertiary centers.

Migraine associated vertigo covers a significant percentage of vertigo cases in many studies. Neurological examination is normal. A study by Langhagen et al. (13) including 147 children with vertigo also showed that the most common cause of vertigo was MAV under the age of 12 years and psychogenic vertigo in children older than 12 years. Erbek et al. (14) studied 50 children with vertigo and showed that the main symptoms accompanying vertigo were headache, nausea, and vomiting. In two large-scale studies reported earlier, it was found that headache frequently accompanied vertigo in children $(15,16)$. In our study, the most common diagnosis of migraine group in which headache accompanied vertigo was MAV ( $\mathrm{p}<0.001)$. Headache being the most common symptom accompanying vertigo (57.5\%) indicates the need for questioning about the presence of vertigo in patients presenting with headache. In this study, a higher number of patients with MAV than those with BCPV was related to a greater mean age of the enrolled patients (12 \pm 4 years). Early childhood migraine variants

Benign childhood paroxysmal vertigo is highly associated migraine and it is important in pre-school age group. Childhood migraine related disorders or equivalents (BCPV, cyclic vomiting syndrome etc.) are the most common episodic disorders of pediatric age group $(3,9,17,18)$. Zhang et al. (17) showed that $26(46.4 \%)$ of 56 BCPV patients had a family history of migraine. Marcelli et al. (18) in a pediatric study, compared eight children with benign paroxysmal positional vertigo (BPPV) and 10 children with BCPV and found that all patients with BCPV had a family history of migraine whereas none of those with BPPV had such a family history. In line with previous studies in the literature, our study found a higher rate of BCPV under the age of 6 , with patients having a high percentage of family history of migraine. However, attack duration was significantly shorter than in the other groups.

Orthostatic hypotension is particularly seen in children of adolescence. Pediatric $\mathrm{OH}$ was reported by Zhao et al., and they described that most children with $\mathrm{OH}$ were in adolescence age group and presented vertigo (46.9\%) and syncope as their main clinical manifestations, which were often induced by sudden postural changes or prolonged standing. American Autonomic Society has defined orthostatic hypotension as a persistent drop of systolic/diastolic blood pressure of more than 20/10 $\mathrm{mmHg}$ without dizziness or heart rate increase, which occurs when a person stands in upright position without moving arms and legs for 3 minutes (19-21). Riina et al. reported this condition in 4 of 119 patients (22). In our study, on the other hand, 3 patients were found to have $\mathrm{OH}$-associated vertigo. A short duration of vertigo, and inclusion of critical statements such as sudden standing up in a patient's history alone are diagnostic in most cases.

Gruber et al. (23) reported that the symptoms are relieved at follow-up in 50\% of patients with psychogenic vertigo. Langhagen et al. (13) detected an underlying psychiatric disorder in $6(28 \%)$ of 21 patients with psychogenic vertigo. Jahn et al. (24) advocated that specific approaches are required for psychogenic vertigo, the most frequent diagnosis in adolescent girls. Our study found that its prevalence increased above 12 years of age among patients with psychogenic vertigo, and it was more common in girls. This suggested that it should be considered as an etiology in patients presenting with vertigo, particularly in adolescent girls. Also in our study, four $(33.3 \%)$ of the patients with psychogenic vertigo had a comorbid psychiatric disorder (somatization disorder, attention deficit and hyperactivity disorder, obsessive compulsive disorder, bulumia nervosa). In our study group, vertigo was recovered at follow-up in 9 patients with psychogenic vertigo.

This study primarily concluded that vertigo of childhood peaked in two periods, 2-6 years and adolescent age, and we should consider different diagnoses in different age groups. A detailed physical examination, audiological evaluation, and most importantly, a detailed patient history sufficient to reveal the cause of vertigo in most patients. While the most common clinical cause of vertigo in children aged six years or younger was BCPV, psychogenic vertigo was a more prominent diagnosis in those older than 6 years. A review of previous studies indicates that the most common causes of vertigo in children have been variably reported. This difference was considered to stem basically from the differences of study inclusion criteria and the differences of patient profiles based on the study setting (such as Pediatric Neurology department or Otorhinolaryngology department). A limitation of our study was its small sample size, which could have been increased by evaluating patients with different vertigo etiologies followed by other clinics together. As the number of studies investigating vertigo in broad age groups in childhood is too small, we believe that our study would likely contribute to the literature. 


\section{ETHICAL DECLARATIONS}

Ethics Committee Approval: Approval for the study was granted by the Ethics Committee of Kahramanmaraş Sütçü İmam University Medical Faculty (Date: 15.03.2017, session no: 2017/04, Decision No: 43).

Informed Consent: Because the study was designed retrospectively, no written informed consent form was obtained from patients.

Referee Evaluation Process: Externally peer-reviewed.

Conflict of Interest Statement: The authors have no conflicts of interest to declare.

Financial Disclosure: The authors declared that this study has received no financial support.

Author Contributions: All of the authors declare that they have all participated in the design, execution, and analysis of the paper, and that they have approved the final version.

\section{REFERENCES}

1. Jahn K, Langhagen T, Schroeder AS, Heinen F. Vertigo and dizziness in childhood Update on diagnosis and treatment. Neuropediatrics 2011; 42: 129-4.

2. Davitt M, Delvecchio MT, Aronoff SC. The differential diagnosis of vertigo in children: a systematic review of 2726 cases. Pediatr Emerg Care 2020; 36: 368-71.

3. Batu ED, Anlar B, Topçu M, Turanlı G, Aysun S. Vertigo in childhood: a retrospective series of 100 children. Eur J Paediatr Neurol 2015; 19: 226-32.

4. Raucci U, Vanacore N, Paolino MC, et al. Vertigo/dizziness in pediatric emergency department: Five years' experience. Cephalalgia 2016; 36: 593-8.

5. Gümüşçü F, Benli ED, Demirtaş S, Karahan O. A case with late clinical presentation of Takayasu's arteritis. Dicle Tip Derg 2014; 41: 421-4.

6. Headache Classification Committee of the International Headache Society (IHS). The international classification of Headache disorders, 3rd edition (beta version). Cephalalgia 2013; 33: 629-808.

7. Furman JM, Balaban CD. Vestibular migraine. Ann N Y Acad Sci 2015; 1343: 90-6.

8. Stahl JS, Daroff RB. Time for more attention to migrainous vertigo? Neurology 2001; 56: 428-9.

9. Akdak G. Benign paroxysmal vertigo of childhood. in: headache in children and adolescents. $1^{\text {st }}$ ed. Abu-Arefeh A, Özge A eds. Springer (Switzerland) 2016; pp: 129-34.

10. Furman JM, Marcus DA, Balaban CD. Migrainous vertigo: development of a pathogenetic model and structured diagnostic interview. Current Opinion in Neurology 2003; 16: 5-13.

11. Lempert T, Neuhauser H, Daroff RB. Vertigo as a symptom of migraine. Ann N Y Acad Sci 2009; 1164: 242-51.

12. Casani AP, Dallan I, Navari E, Sellari Franceschini S, Cerchiai N. Vertigo in childhood: proposal for a diagnostic algorithm based upon clinical experience. Acta Otorhinolaryngol Ital 2015; 35: 180-5.

13. Langhagen T, Schroeder AS, Rettinger N, Borggraefe I, Jahn K. Migraine-related vertigo and somatoform vertigo frequently occur in children and are often associated. Neuropediatrics 2013; 44: 55-8.
14. Erbek SH, Erbek SS, Yilmaz I, et al. Vertigo in childhood: a clinical experience. Int J Pediatr Otorhinolaryngol 2006; 70: 1547-54.

15. Balatsouras DG, Kaberos A, Assimakopoulos D, Katotomichelakis M, Economou NC, Korres SG. Etiology of vertigo in children. Int J Pediatr Otorhinolaryngol 2007; 71: 487-94.

16. Humphriss RL, Hall AJ. Dizziness in 10-year-old children: an epidemiological study. Int J Pediatr Otorhinolaryngol 2011; 75: 395-400.

17. Zhang D, Fan Z, Han Y, et al. Benign paroxysmal vertigo of childhood: Diagnostic value of vestibular test and high stimulus rate auditory brainstem response test. Int J Pediatr Otorhinolaryngol 2012; 76: 107-10.

18. Marcelli V, Piazza F, Pisani F, Marciano E. Neuro-otological features of benign paroxysmal vertigo and benign paroxysmal positioning vertigo in children: A follow-up study. Brain Dev 2006; 28: 80-4.

19. Stewart MJ. Autonomic nervous system dysfunction in adolescents with postural orthostatic tachycardia syndrome and chronic fatigue syndrome is characterized by attenuated vagal baroreflex and potentiated sympathetic vasomotion. Pediatr Res 2000; 48: 218-26.

20. The Consensus Committee of the American Autonomic Society and the American Academy of Neurology. Consensus statement on the definition of orthostatic hypotension, pure autonomic failure, and multiple system atrophy. Neurology 1996; 46: 1470.

21. Zhao J, Yang J, Jin H, Du J. Clinical analysis of orthostatic hypertensin in children. Clin J Pediatr 2012; 50: 839-42.

22. Riina N, Ilmari P, Kentala E. Vertigo and imbalance in children: a retrospective study in a Helsinki University Otorhinolaryngology Clinic. Arch Otolaryngol Head Neck Surg 2005; 131: 996-1000.

23. Gruber M, Cohen-Kerem R, Kaminer M, Shupak A. Vertigo in children and adolescents: characteristics and outcome. ScientificWorldJournal 2012; 2012: 109624.

24. Jahn K, Langhagen T, Heinen F. Vertigo and dizziness in children. Curr Opin Neurol 2015; 28: 78-82. 